\title{
A New Difference Sequence Set of Order $\alpha$ and Its Geometrical Properties
}

\author{
Mikail Et ${ }^{1}$ and Vatan Karakaya ${ }^{2}$ \\ ${ }^{1}$ Department of Mathematics, Firat University, 23119, Elaž̆, Turkey \\ ${ }^{2}$ Department of Mathematical Engineering, Yildiz Technical University, Davutpasa Campus, Esenler, 34750 Istanbul, Turkey \\ Correspondence should be addressed to Vatan Karakaya; vkkaya@yildiz.edu.tr
}

Received 15 June 2014; Accepted 19 August 2014; Published 11 September 2014

Academic Editor: Cristina Pignotti

Copyright (c) 2014 M. Et and V. Karakaya. This is an open access article distributed under the Creative Commons Attribution License, which permits unrestricted use, distribution, and reproduction in any medium, provided the original work is properly cited.

We introduce a new class of sequences named as $m_{\alpha}\left(\Delta^{r}, \phi, p\right)$ and, for this space, we study some inclusion relations, topological properties, and geometrical properties such as order continuous, the Fatou property, and the Banach-Saks property of type $p$.

\section{Introduction, Definitions, and Preliminaries}

By $w$ we denote the space of all complex (or real) sequences. If $x \in w$, then we simply write $x=\left(x_{k}\right)$ instead of $x=\left(x_{k}\right)_{k=0}^{\infty}$. We will write $\ell_{\infty}, c$, and $c_{0}$ for the sequence spaces of all bounded, convergent, and null sequences, respectively. Also by $\ell_{1}$ and $\ell_{p}$ we denote the spaces of all absolutely summable and $p$-absolutely summable series, respectively.

The notion of difference sequence spaces was generalized by Et and Colak [1] such as $X\left(\Delta^{r}\right)=\left\{x=\left(x_{k}\right): \Delta^{r} x \in X\right\}$, for $X=\ell_{\infty}, c$, and $c_{0}$. They showed that these sequence spaces are $B K$-spaces with the norm

$$
\|x\|_{\Delta}=\sum_{i=1}^{r}\left|x_{i}\right|+\left\|\Delta^{r} x\right\|_{\infty}
$$

where $r \in \mathbb{N}, \Delta^{0} x=x, \Delta x=\left(x_{k}-x_{k+1}\right), \Delta^{r} x=\left(\Delta^{r} x_{k}\right)=$ $\left(\Delta^{r-1} x_{k}-\Delta^{r-1} x_{k+1}\right)$, and $\Delta^{r} x_{k}=\sum_{v=0}^{r}(-1)^{v}\left(\begin{array}{c}r \\ v\end{array}\right) x_{k+v}$. Recently difference sequences and related concepts have been studied in ([2-13]) and by many others.

Let $E$ be a sequence space. Then $E$ is called

(i) solid (or normal) if $\left(\alpha_{k} x_{k}\right) \in E$ for all sequences $\left(\alpha_{k}\right)$ of scalars with $\left|\alpha_{k}\right| \leq 1$ for all $k \in \mathbb{N}$, whenever $\left(x_{k}\right) \in E$,

(ii) symmetric if $\left(x_{k}\right) \in E$ implies $\left(x_{\pi(k)}\right) \in E$, where $\pi$ is a permutation of $\mathbb{N}$, (iii) monotone provided $E$ contains the canaonical preimages of all its step spaces,

(iv) sequence algebra if $x \cdot y \in E$, whenever $x, y \in E$.

It is well known that if $E$ is normal then $E$ is monotone.

Throughout this paper $\varphi_{s}$ denotes the class of all subsets of $\mathbb{N}$; those do not contain more than $s$ elements. Let $\left(\phi_{n}\right)$ be a nondecreasing sequence of positive numbers such that $n \phi_{n+1} \leq(n+1) \phi_{n}$ for all $n \in \mathbb{N}$. The class of all sequences $\left(\phi_{n}\right)$ is denoted by $\Phi$. The sequence space $m(\phi)$ was introduced by Sargent [14] and he studied some of its properties and obtained some relations with the space $\ell_{p}$. Later on it was investigated by Tripathy and Sen [15] and Tripathy and Mahanta [16].

Let us recall that a sequence $\{v(i)\}_{i=1}^{\infty}$ in a Banach space $X$ is called Schauder basis of $X$ (or basis for short) if for each $x \in X$ there exists a unique sequence $\{\lambda(i)\}_{i=1}^{\infty}$ of scalars such that $x=\sum_{i=1}^{\infty} \lambda(i) v(i)$; that is, $\lim _{n \rightarrow \infty} \sum_{i=1}^{n} \lambda(i) v(i)=x$.

A sequence space $X$ with a linear topology is called a $K$ space if each of the projection maps $P_{i}: X \rightarrow \mathbb{C}$ defined by $P_{i}(x)=x(i)$ for $x=(x(i))_{i=1}^{\infty} \in X$ is continuous for each natural $i$. A Fréchet space is a complete metric linear space and the metric is generated by an $F$-norm and a Fréchet space which is a $K$-space is called an $F K$-space; that is, a $K$-space $X$ is called an $F K$-space if $X$ is a complete linear metric space. In other words, $X$ is an $F K$-space if $X$ is a Fréchet space with 
continuous coordinate projections. All the sequence spaces mentioned above are $F K$ spaces except the space $c_{00}$.

An $F K$-space $X$ which contains the space $c_{00}$ is said to have the property $A K$ if for every sequence $\{x(i)\} \in X, x=$ $\sum_{i=1}^{\infty} x(i) e(i)$ where $e(i)=(0,0, \ldots, 1$ (ith-place $\left.), 0,0, \ldots\right)$.

A Banach space $X$ is said to be a Köthe sequence space (see $[17,18])$ if $X$ is a subspace of $w$ such that

(i) if $x \in w, y \in X$ and $|x(i)| \leq|y(i)|$ for all $i \in \mathbb{N}$, then $x \in X$ and $\|x\| \leq\|y\|$

(ii) there exists an element $x \in X$ such that $x(i)>0$ for all $i \in \mathbb{N}$.

We say that $x \in X$ is order continuous if for any sequence $\left(x_{n}\right)$ in $X$ such that $x_{n}(i) \leq|x(i)|$ for each $i \in \mathbb{N}$ and $x_{n}(i) \rightarrow 0$ $(n \rightarrow \infty)$ we have that $\left\|x_{n}\right\| \rightarrow 0$ holds.

A Köthe sequence space $X$ is said to be order continuous if all sequences in $X$ are order continuous. It is easy to see that $x \in X$ is order continuous if and only if $\|(0,0, \ldots, 0, x(n+$ $1), x(n+2), \ldots) \| \rightarrow 0$ as $n \rightarrow \infty$.

A Köthe sequence space $X$ is said to have the Fatou property if, for any real sequence $x$ and any $\left\{x_{n}\right\}$ in $X$ such that $x_{n} \uparrow x$ coordinatewisely and $\sup _{n}\left\|x_{n}\right\|<\infty$, we have that $x \in X$ and $\left\|x_{n}\right\| \rightarrow\|x\|$.

A Banach space $X$ is said to have the BanachSaks property if every bounded sequence $\left\{x_{n}\right\}$ in $X$ admits a subsequence $\left\{z_{n}\right\}$ such that the sequence $\left\{t_{k}(z)\right\}$ is convergent in $X$ with respect to the norm, where

$$
t_{k}(z)=\frac{1}{k}\left(z_{1}+z_{2}+\cdots+z_{k}\right), \quad \forall k \in \mathbb{N}
$$

Some of recent works on geometric properties of sequence space can be found in the following list ([19-21]).

\section{Inclusion and Topological Properties of the Space $m_{\alpha}\left(\Delta^{r}, \phi, p\right)$}

In this section we introduce a new class of sequences and establish some inclusion relations. Also we show that this space is not perfect and normal.

Let $r$ be a fixed positive integer, $\alpha \in(0,1]$ any real number, and $p$ a positive real number such that $1 \leq p<\infty$. Now we define the sequence space $m_{\alpha}\left(\Delta^{r}, \phi, p\right)$ as

$$
m_{\alpha}\left(\Delta^{r}, \phi, p\right)=\left\{x \in w: \sup _{s \geq 1, \sigma \in \varphi_{s}} \frac{1}{\phi_{s}^{\alpha}} \sum_{n \in \sigma}\left|\Delta^{r} x_{n}\right|^{p}<\infty\right\} .
$$

In the case $p=1$, we will write $m_{\alpha}\left(\Delta^{r}, \phi\right)$ instead of $m_{\alpha}\left(\Delta^{r}, \phi, p\right)$ and in the special case $m=0$ and $p=1$ we will write $m_{\alpha}(\phi)$ instead of $m_{\alpha}\left(\Delta^{r}, \phi, p\right)$.

The proof of each of the following results is straightforward, so we choose to state these results without proof.

Theorem 1. Let $\phi \in \Phi, \alpha \in(0,1]$, and let $p$ be a positive real number such that $1 \leq p<\infty$. Then the sequence space $m_{\alpha}\left(\Delta^{r}, \phi, p\right)$ is a BK-space normed by

$$
\|x\|=\sum_{i=1}^{r}\left|x_{i}\right|+\sup _{s \geq 1, \sigma \in \varphi_{s}} \frac{1}{\phi_{s}^{\alpha}}\left(\sum_{n \in \sigma}\left|\Delta^{r} x_{n}\right|^{p}\right)^{1 / p} .
$$

Theorem 2. Let $\phi \in \Phi, \alpha \in(0,1]$, and let $p$ be a positive real number such that $1 \leq p<\infty$; then $m_{\alpha}\left(\Delta^{r}, \phi\right) \subset m_{\alpha}\left(\Delta^{r}, \phi, p\right)$.

Theorem 3. Let $\alpha$ and $\beta$ be fixed real numbers such that $0<$ $\alpha \leq \beta \leq 1$ and $p$ a positive real number such that $1 \leq p<\infty$; then $m_{\alpha}\left(\Delta^{r}, \phi, p\right) \subset m_{\beta}\left(\Delta^{r}, \phi, p\right)$.

Theorem 4. Let $\alpha$ and $\beta$ be fixed real numbers such that $0<$ $\alpha \leq \beta \leq 1$ and $p$ a positive real number such that $1 \leq p<$ $\infty$. For any two sequences $\left(\phi_{s}\right)$ and $\left(\psi_{s}\right)$ of real numbers such that $\phi, \psi \in \Phi$. Then $m_{\alpha}\left(\Delta^{r}, \phi, p\right) \subset m_{\beta}\left(\Delta^{r}, \psi, p\right)$ if and only if $\sup _{s \geq 1}\left(\phi_{s}^{\alpha} / \psi_{s}^{\beta}\right)<\infty$.

Proof. Let $x \in m_{\alpha}\left(\Delta^{r}, \phi, p\right)$ and $\sup _{s \geq 1}\left(\phi_{s}^{\alpha} / \psi_{s}^{\beta}\right)<\infty$. Then

$$
\sup _{s \geq 1, \sigma \in \varphi_{s}} \frac{1}{\phi_{s}^{\alpha}} \sum_{n \in \sigma}\left|\Delta^{r} x_{n}\right|^{p}<\infty
$$

and there exists a positive number $K$ such that $\phi_{s}^{\alpha} \leq K \psi_{s}^{\beta}$ and so that $1 / \psi_{s}^{\beta} \leq K / \phi_{s}^{\alpha}$ for all $s$. Therefore for all $s$ we have

$$
\frac{1}{\psi_{s}^{\beta}} \sum_{n \in \sigma}\left|\Delta^{r} x_{n}\right|^{p} \leq \frac{K}{\phi_{s}^{\alpha}} \sum_{n \in \sigma}\left|\Delta^{r} x_{n}\right|^{p} .
$$

Now taking supremum over $s \geq 1$ and $\sigma \in \varphi_{s}$ we get

$$
\sup _{s \geq 1, \sigma \in \varphi_{s}} \frac{1}{\psi_{s}^{\beta}} \sum_{n \in \sigma}\left|\Delta^{r} x_{n}\right|^{p} \leq \sup _{s \geq 1, \sigma \in \varphi_{s}} \frac{K}{\phi_{s}^{\alpha}} \sum_{n \in \sigma}\left|\Delta^{r} x_{n}\right|^{p}
$$

and so $x \in m_{\beta}\left(\Delta^{r}, \psi, p\right)$.

Conversely let $m_{\alpha}\left(\Delta^{r}, \phi, p\right) \subset m_{\beta}\left(\Delta^{r}, \psi, p\right)$ and suppose that $\sup _{s \geq 1}\left(\phi_{s}^{\alpha} / \psi_{s}^{\beta}\right)=\infty$. Then there exists an increasing sequence $\left(s_{i}\right)$ of naturals numbers such that $\lim _{i}\left(\phi_{s_{i}}^{\alpha} / \psi_{s_{i}}^{\beta}\right)=$ $\infty$. Let $B \in \mathbb{R}^{+}$, where $\mathbb{R}^{+}$is the set of positive real numbers; then there exists $i_{0} \in \mathbb{N}$ such that $\phi_{s_{i}}^{\alpha} / \psi_{s_{i}}^{\beta}>B$ for all $s_{i} \geq i_{0}$. Hence $\phi_{s_{i}}^{\alpha}>B \psi_{s_{i}}^{\beta}$ and so $1 / \psi_{s_{i}}^{\beta}>B / \phi_{s_{i}}^{\alpha}$. Then we can write

$$
\frac{1}{\psi_{s_{i}}^{\beta}} \sum_{n \in \sigma}\left|\Delta^{r} x_{n}\right|^{p}>\frac{B}{\phi_{s_{i}}^{\alpha}} \sum_{n \in \sigma}\left|\Delta^{r} x_{n}\right|^{p}
$$

for all $s_{i} \geq i_{0}$. Now taking supremum over $s_{i} \geq i_{0}$ and $\sigma \in \varphi_{s}$ we get

$$
\sup _{s_{i} \geq i_{0}, \sigma \in \varphi_{s}} \frac{1}{\psi_{s_{i}}^{\beta}} \sum_{n \in \sigma}\left|\Delta^{r} x_{n}\right|^{p}>\sup _{s_{i} \geq i_{0}, \sigma \in \varphi_{s}} \frac{B}{\phi_{s_{i}}^{\alpha}} \sum_{n \in \sigma}\left|\Delta^{r} x_{n}\right|^{p} .
$$

Since (9) holds for all $B \in \mathbb{R}^{+}$(we may take the number $B$ sufficiently large), we have

$$
\sup _{s_{i} \geq i_{0}, \sigma \in \varphi_{s}} \frac{1}{\psi_{s_{i}}^{\beta}} \sum_{n \in \sigma}\left|\Delta^{r} x_{n}\right|^{p}=\infty
$$

when $x \in m_{\alpha}\left(\Delta^{r}, \phi, p\right)$ with

$$
0<\sup _{s \geq 1, \sigma \in \varphi_{s}} \frac{1}{\phi_{s}^{\alpha}} \sum_{n \in \sigma}\left|\Delta^{r} x_{n}\right|^{p}<\infty .
$$

Hence $x \notin m_{\beta}\left(\Delta^{r}, \psi, p\right)$. This contradicts to $m_{\alpha}\left(\Delta^{r}, \phi, p\right) \subset$ $m_{\beta}\left(\Delta^{r}, \psi, p\right)$. Hence $\sup _{s \geq 1}\left(\phi_{s}^{\alpha} / \psi_{s}^{\beta}\right)<\infty$. 

4

The following results are derivable easily from Theorem

Corollary 5. Let $\alpha$ and $\beta$ be fixed real numbers such that $0<$ $\alpha \leq \beta \leq 1$ and $p$ a positive real number such that $1 \leq p<\infty$. For any two sequences $\left(\phi_{s}\right)$ and $\left(\psi_{s}\right)$ of real numbers such that $\phi, \psi \in \Phi$. Then one has

(i) $m_{\alpha}\left(\Delta^{r}, \phi, p\right)=m_{\beta}\left(\Delta^{r}, \psi, p\right)$ if and only if $0<$ $\inf _{s \geq 1}\left(\phi_{s}^{\alpha} / \psi_{s}^{\beta}\right)<\sup _{s \geq 1}\left(\phi_{s}^{\alpha} / \psi_{s}^{\beta}\right)<\infty$,

(ii) $m_{\alpha}\left(\Delta^{r}, \phi, p\right)=m_{\alpha}\left(\Delta^{r}, \psi, p\right)$ if and only if $0<$ $\inf _{s \geq 1}\left(\phi_{s}^{\alpha} / \psi_{s}^{\alpha}\right)<\sup _{s \geq 1}\left(\phi_{s}^{\alpha} / \psi_{s}^{\alpha}\right)<\infty$,

(iii) $m_{\alpha}\left(\Delta^{r}, \phi, p\right)=m_{\beta}\left(\Delta^{r}, \phi, p\right)$ if and only if $0<$ $\inf _{s \geq 1}\left(\phi_{s}^{\alpha} / \phi_{s}^{\beta}\right)<\sup _{s \geq 1}\left(\phi_{s}^{\alpha} / \phi_{s}^{\beta}\right)<\infty$.

Theorem 6. Consider $m_{\alpha}\left(\Delta^{r-1}, \phi, p\right) \subset m_{\alpha}\left(\Delta^{r}, \phi, p\right)$ and the inclusion is strict.

Proof. It follows from Minkowski's inequality. To show the inclusion is strict, let $\phi_{n}=1$ for all $n \in \mathbb{N}, \alpha=1, p=1$, and $x=\left(k^{r-1}\right)$; then $x \in m_{\alpha}\left(\Delta^{r}, \phi, p\right) \backslash m_{\alpha}\left(\Delta^{r-1}, \phi, p\right)$.

Theorem 7. The sequence space $m_{\alpha}(\phi)$ is solid and hence monotone, but the sequence space $m_{\alpha}\left(\Delta^{r}, \phi, p\right)$ is neither solid nor symmetric and sequence algebra for $m \geq 1$.

Proof. Let $x \in m_{\alpha}(\phi)$ and $y=\left(y_{n}\right)$ be sequences such that $\left|x_{n}\right| \leq\left|y_{n}\right|$ for each $n \in \mathbb{N}$. Then we get

$$
\sup _{s \geq 1, \sigma \in \varphi_{s}} \frac{1}{\phi_{s}^{\alpha}} \sum_{n \in \sigma}\left|x_{n}\right| \leq \sup _{s \geq 1, \sigma \in \varphi_{s}} \frac{1}{\phi_{s}^{\alpha}} \sum_{n \in \sigma}\left|y_{n}\right| .
$$

Hence $m_{\alpha}(\phi)$ is solid and hence monotone. To show the space $m_{\alpha}\left(\Delta^{r}, \phi, p\right)$ is normal, let $\phi_{n}=1$, for all $n \in \mathbb{N}$, $\alpha=1, p=1$, and $x=\left(k^{r-1}\right)$, then $x \in m_{\alpha}\left(\Delta^{r}, \phi, p\right)$; but $\left(\alpha_{k} x_{k}\right) \notin m_{\alpha}\left(\Delta^{r}, \phi, p\right)$ when $\alpha_{k}=(-1)^{k}$ for all $k \in \mathbb{N}$. Hence $m_{\alpha}\left(\Delta^{r}, \phi, p\right)$ is not solid. The other cases can be proved on considering similar examples.

Theorem 8. Consider

$$
\ell_{p}\left(\Delta^{r}\right) \subset m_{\alpha}\left(\Delta^{r}, \phi, p\right) \subset \ell_{\infty}\left(\Delta^{r}\right) .
$$

Proof. It is omitted.

Theorem 9. If $0<p<q$, then $m_{\alpha}\left(\Delta^{r}, \phi, p\right) \subset m_{\alpha}\left(\Delta^{r}, \phi, q\right)$.

Proof. Proof follows from the following inequality:

$$
\left(\sum_{k=1}^{n}\left|x_{k}\right|^{q}\right)^{1 / q}<\left(\sum_{k=1}^{n}\left|x_{k}\right|^{q}\right)^{1 / q}, \quad(0<p<q) .
$$

\section{Geometrical Properties of the Space $m_{\alpha}\left(\Delta^{r}, \phi, p\right)$}

In this section, we study some geometrical properties of the space $m_{\alpha}\left(\Delta^{r}, \phi, p\right)$. Some of these geometrical properties are the order continuous, the Fatou property, and the BanachSaks property of type $p$. Let us start with the following theorem.

Theorem 10. The space $m_{\alpha}\left(\Delta^{r}, \phi, p\right)$ is order continuous.

Proof. To prove this theorem, we have to show that $m_{\alpha}\left(\Delta^{r}\right.$, $\phi, p)$ is an $A K$-space. It is easy to see that $m_{\alpha}\left(\Delta^{r}, \phi, p\right)$ contains $c_{00}$ which is the space of real sequences which have only a finite number of nonzero coordinates. By using definition of $A K$-properties, we have that $x=\{x(i)\} \in$ $m_{\alpha}\left(\Delta^{r}, \phi, p\right)$ has a unique representation $x=\sum_{i=1}^{\infty} x(i) e(i)$; that is, $\left\|x-x^{[j]}\right\|_{m_{\alpha}\left(\Delta^{r}, \phi, p\right)}=\|(0,0, \ldots, x(j), x(j+1)$, $\ldots) \|_{m_{\alpha}\left(\Delta^{r}, \phi, p\right)} \rightarrow 0$ as $j \rightarrow \infty$, which means that $m_{\alpha}\left(\Delta^{r}, \phi\right.$, $p)$ has $A K$. Hence, since $B K$-space $m_{\alpha}\left(\Delta^{r}, \phi, p\right)$ containing $c_{00}$ has $A K$-property, the space $m_{\alpha}\left(\Delta^{r}, \phi, p\right)$ is order continuous.

Theorem 11. The space $m_{\alpha}\left(\Delta^{r}, \phi, p\right)$ has the Fatou property.

Proof. Let $x$ be any real sequence from $(w)_{+}$and $\left\{x_{n}\right\}$ any nondecreasing sequence of nonnegative elements from $m_{\alpha}\left(\Delta^{r}, \phi, p\right)$ such that $x_{n}(i) \rightarrow x(i)$ as $n \rightarrow \infty$ coordinatewisely and $\sup _{n}\left\|x_{n}\right\|_{m_{\alpha}\left(\Delta^{r}, \phi, p\right)}<\infty$.

Let us denote $\tau=\sup _{n}\left\|x_{n}\right\|_{m_{\alpha}\left(\Delta^{r}, \phi, p\right)}$. Then, since the supremum is homogeneous, we have

$$
\begin{gathered}
\frac{1}{\tau}\left(\sum_{i=1}^{r}\left|x_{n}(i)\right|+\sup _{s \geq 1, \sigma \in \varphi_{s}} \frac{1}{\phi_{s}^{\alpha}}\left(\sum_{i \in \sigma}\left|\Delta^{r} x_{n}(i)\right|^{p}\right)^{1 / p}\right) \\
=\sum_{i=1}^{r}\left|\frac{x_{n}(i)}{\tau}\right|+\sup _{s \geq 1, \sigma \in \varphi_{s}} \frac{1}{\phi_{s}^{\alpha}}\left(\sum_{n \in \sigma}\left|\frac{\Delta^{r} x_{n}(i)}{\tau}\right|^{p}\right)^{1 / p} \\
\leq \sum_{i=1}^{r}\left|\frac{x_{n}(i)}{\left\|x_{n}\right\|_{m_{\alpha}\left(\Delta^{r}, \phi, p\right)}}\right| \\
\quad+\sup _{s \geq 1, \sigma \in \varphi_{s}} \frac{1}{\phi_{s}^{\alpha}}\left(\sum_{n \in \sigma}\left|\frac{\Delta^{r} x_{n}(i)}{\left\|x_{n}\right\|_{m_{\alpha}\left(\Delta^{r}, \phi, p\right)}}\right|^{p}\right)^{1 / p} \\
=\frac{1}{\left\|x_{n}\right\|_{m_{\alpha}\left(\Delta^{r}, \phi, p\right)}}\left\|x_{n}\right\|_{m_{\alpha}\left(\Delta^{r}, \phi, p\right)}=1 .
\end{gathered}
$$

Moreover, by the assumptions that $\left\{x_{n}\right\}$ is nondecreasing and convergent to $x$ coordinatewisely and by the Beppo-Levi theorem, we have

$$
\begin{aligned}
\frac{1}{\tau} \lim _{n \rightarrow \infty}\left(\sum_{i=1}^{r}\left|x_{n}(i)\right|+\sup _{s \geq 1, \sigma \in \varphi_{s}} \frac{1}{\phi_{s}^{\alpha}}\left(\sum_{i \in \sigma}\left|\Delta^{r} x_{n}(i)\right|^{p}\right)^{1 / p}\right) \\
=\sum_{i=1}^{r}\left|\frac{x(i)}{\tau}\right|+\sup _{s \geq 1, \sigma \in \varphi_{s}} \frac{1}{\phi_{s}^{\alpha}}\left(\sum_{i \in \sigma}\left|\frac{\Delta^{r} x(i)}{\tau}\right|^{p}\right)^{1 / p} \\
=\left\|\frac{x}{s}\right\|_{\left.m_{\alpha}\left(\Delta^{r}, \phi, p\right)\right)} \leq 1,
\end{aligned}
$$


whence

$$
\begin{aligned}
\|x\|_{\left.\left.m_{\alpha}\left(\Delta^{r}, \phi, p\right)\right)\right)} & \leq \tau=\sup _{n}\left\|x_{n}\right\|_{\left.m_{\alpha}\left(\Delta^{r}, \phi, p\right)\right)} \\
& =\lim _{n \rightarrow \infty}\left\|x_{n}\right\|_{\left.m_{\alpha}\left(\Delta^{r}, \phi, p\right)\right)}<\infty .
\end{aligned}
$$

Therefore, $\left.x \in m_{\alpha}\left(\Delta^{r}, \phi, p\right)\right)$. On the other hand, since $0 \leq x_{n} \leq x$ for any natural number $n$ and the sequence $\left\{x_{n}\right\}$ is nondecreasing, we obtain that the sequence $\left\{\left\|x_{n}\right\|_{\left.m_{\alpha}\left(\Delta^{r}, \phi, p\right)\right)}\right\}$ is bounded from above by $\|x\|_{\left.m_{\alpha}\left(\Delta^{r}, \phi, p\right)\right)}$. As a result, $\lim _{n \rightarrow \infty}\left\|x_{n}\right\|_{\left.m_{\alpha}\left(\Delta^{r}, \phi, p\right)\right)} \leq\|x\|_{\left.m_{\alpha}\left(\Delta^{r}, \phi, p\right)\right)}$, which together with the opposite inequality proved already yields that $\|x\|_{\left.m_{\alpha}\left(\Delta^{r}, \phi, p\right)\right)}=\lim _{n}\left\|x_{n}\right\|_{\left.m_{\alpha}\left(\Delta^{r}, \phi, p\right)\right)}$.

Theorem 12. The space $\left.m_{\alpha}\left(\Delta^{r}, \phi, p\right)\right)$ has the Banach-Saks property of the type $p$.

Proof. It can be proved with standard technic.

\section{Conflict of Interests}

The authors declare that there is no conflict of interests regarding the publication of this paper.

\section{References}

[1] M. Et and R. Çolak, "On some generalized difference sequence spaces," Soochow Journal of Mathematics, vol. 21, no. 4, pp. 377386, 1995.

[2] B. Altay and F. Basar, "On the fine spectrum of the difference operator $\Delta$ on $c_{0}$ and and c," Information Science, vol. 168, no. 1-4, pp. 217-224, 2004.

[3] Ç. A. Bektas, M. Et, and R. Çolak, "Generalized difference sequence spaces and their dual spaces," Journal of Mathematical Analysis and Applications, vol. 292, no. 2, pp. 423-432, 2004.

[4] N. L. Braha, "On asymptotically $\Delta^{m}$-lacunary statistical equivalent sequences," Applied Mathematics and Computation, vol. 219, no. 1, pp. 280-288, 2012.

[5] R. Çolak and M. Et, "On some difference sequence sets and their topological properties," Bulletin of the Malaysian Mathematical Sciences Society, vol. 28, no. 2, pp. 125-130, 2005.

[6] M. Et, "Strongly almost summable difference sequences of order $m$ defined by a modulus," Studia Scientiarum Mathematicarum Hungarica, vol. 40, no. 4, pp. 463-476, 2003.

[7] M. Güngor and M. Et, " $\Delta^{r}$-strongly almost summable sequences defined by Orlicz functions," Indian Journal of Pure and Applied Mathematics, vol. 34, no. 8, pp. 1141-1151, 2003.

[8] M. Karakas, M. Et, and V. Karakaya, "Some geometric properties of a new difference sequence space involving lacunary sequences," Acta Mathematica Scientia, vol. 33, no. 6, pp. 17111720, 2013.

[9] M. Mursaleen, R. Çolak, and M. Et, "Some geometric inequalities in a new banach sequence space," Journal of Inequalities and Applications, vol. 2007, Article ID 86757, 6 pages, 2007.

[10] H. Polat, V. Karakaya, and N. Şimşek, "Difference sequence spaces derived by using a generalized weighted mean," Applied Mathematics Letters, vol. 24, no. 5, pp. 608-614, 2011.

[11] B. C. Tripathy, "On a class of difference sequences related to the $p$-normed space $l_{p}$," Demonstratio Mathematica, vol. 36, no. 4 , pp. 867-872, 2003.
[12] S. A. Mohiuddine, K. Raj, and A. Alotaibi, "Some paranormed double difference sequence spaces for Orlicz functions and bounded-regular matrices," Abstract and Applied Analysis, vol. 2014, Article ID 419064, 10 pages, 2014.

[13] S. A. Mohiuddine, K. Raj, and A. Alotaibi, "On some classes of double difference sequences of interval numbers," Abstract and Applied Analysis, vol. 2014, Article ID 516956, 8 pages, 2014.

[14] W. L. C. Sargent, "Some sequence spaces related to the $\mathrm{l}_{p}$ spaces," Journal of the London Mathematical Society. Second Series, vol. 35, pp. 161-171, 1960.

[15] B. C. Tripathy and M. Sen, "On a new class of sequences related to the space $L_{p}$," Tamkang Journal of Mathematics, vol. 33, no. 2, pp. 167-171, 2002.

[16] B. C. Tripathy and S. Mahanta, "On a class of sequences related to the $l_{p}$ space defined by Orlicz functions," Soochow Journal of Mathematics, vol. 29, no. 4, pp. 379-391, 2003.

[17] Y. A. Cui and H. Hudzik, "On the Banach-Saks and weak Banach-Saks properties of some Banach sequence spaces," Acta Scientiarum Mathematicarum, vol. 65, no. 1-2, pp. 179-187, 1999.

[18] J. Diestel, Sequence and Series in Banach Spaces, vol. 92 of Graduate Texts in Mathematics, Springer, Berlin, Germany, 1984.

[19] V. Karakaya and F. Altun, "On some geometric properties of a new paranormed sequence space," Journal of Function Spaces, vol. 2014, Article ID 685382, 8 pages, 2014.

[20] H. Hudzik, V. Karakaya, and M. Mursaleen, "Banach-Saks type and GGurarii modulus of convexity of some Banach sequence spaces," Abstract and Applied Analysis, vol. 2014, Article ID 427382, 9 pages, 2014.

[21] M. Et, M. Karakaş, and V. Karakaya, "Some geometric properties of a new difference sequence space defined by de la ValléePoussin mean," Applied Mathematics and Computation, vol. 234, pp. 237-244, 2014. 


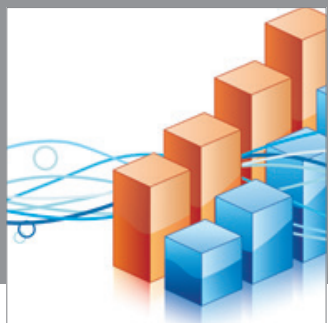

Advances in

Operations Research

mansans

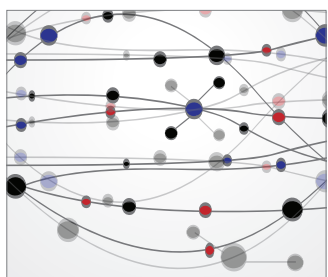

The Scientific World Journal
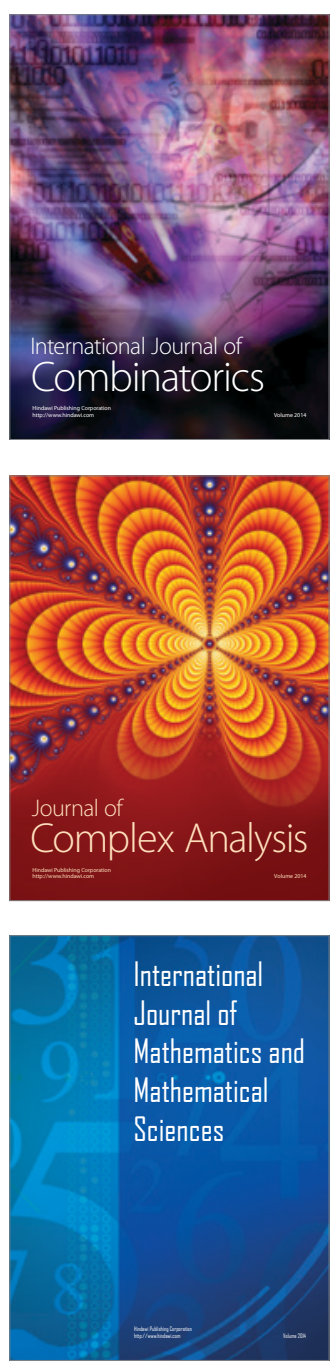
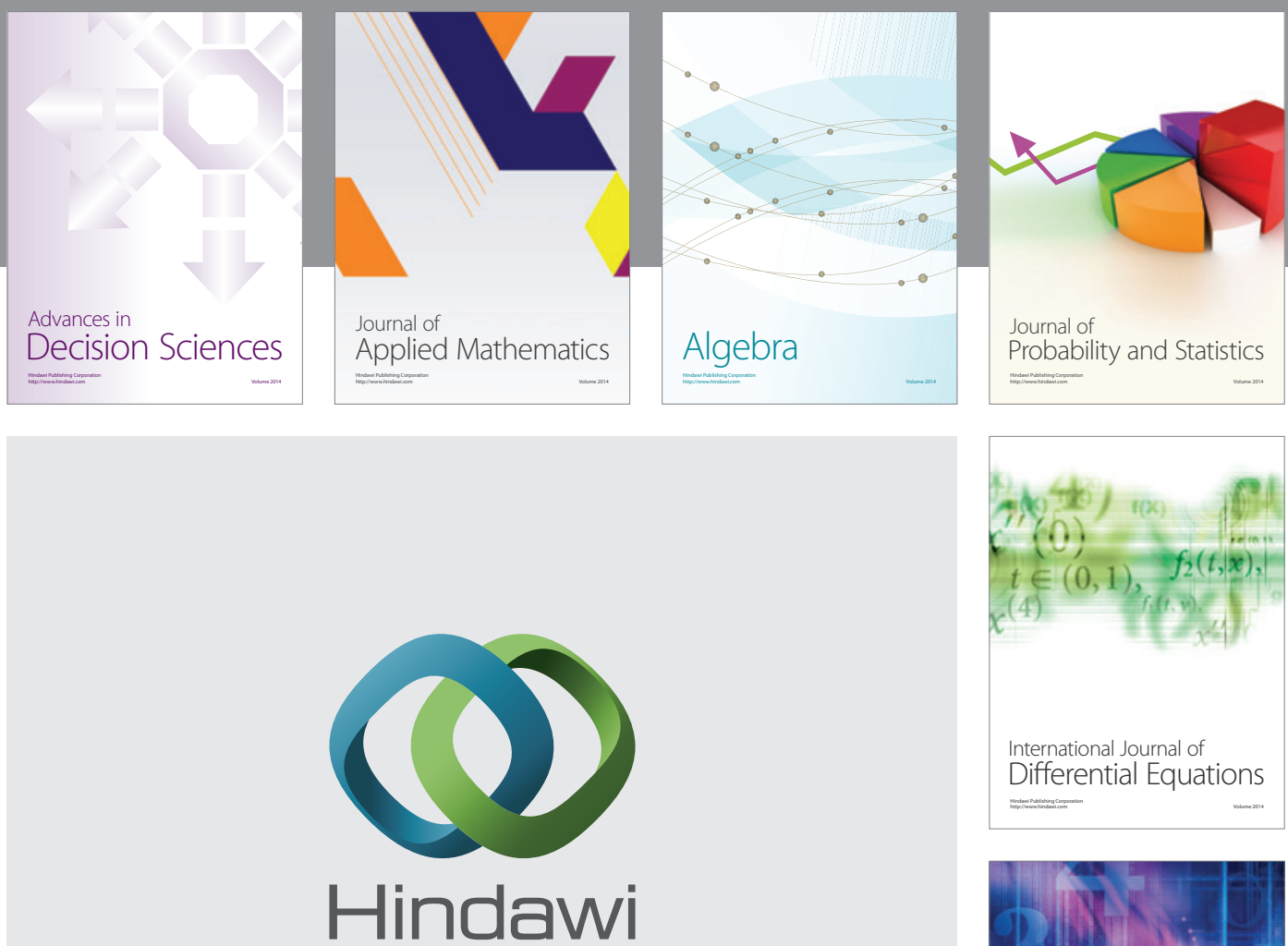

Submit your manuscripts at http://www.hindawi.com
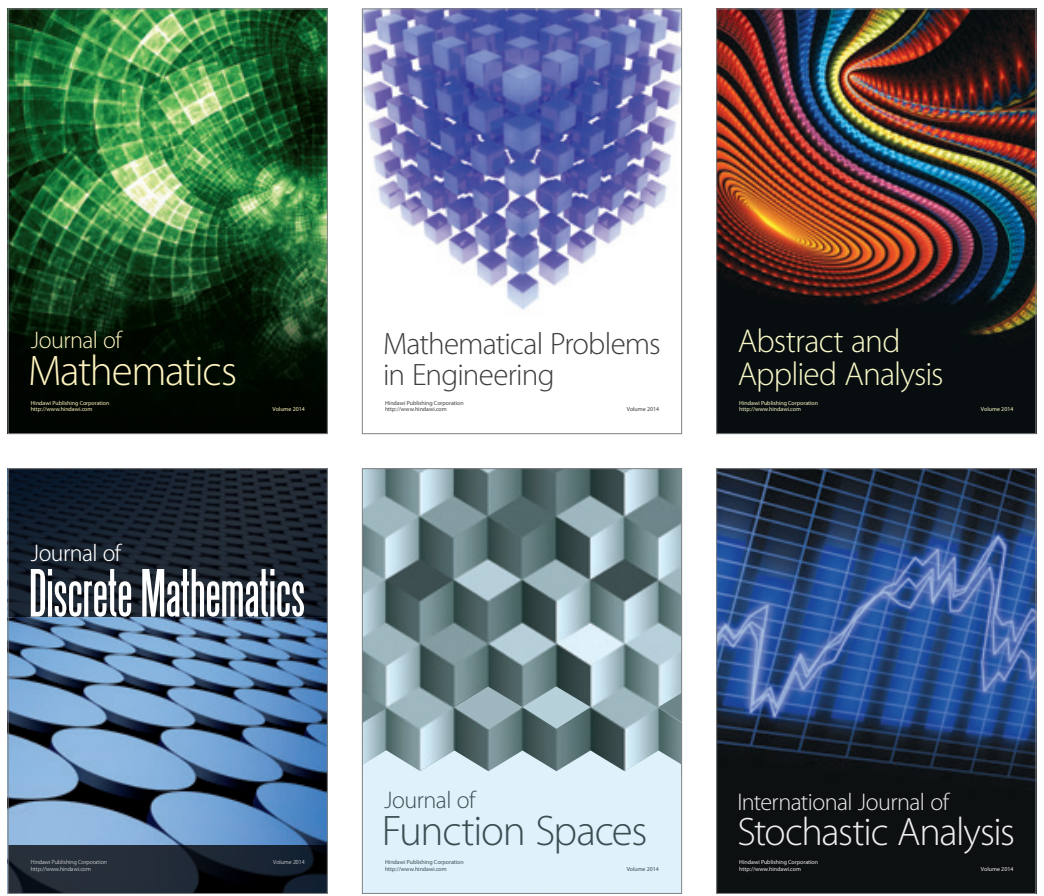

Journal of

Function Spaces

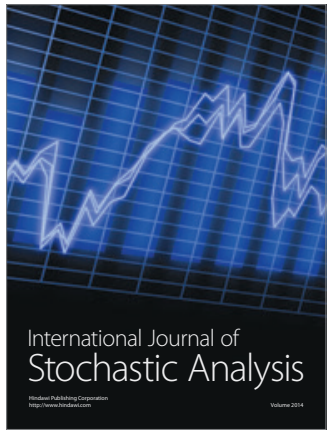

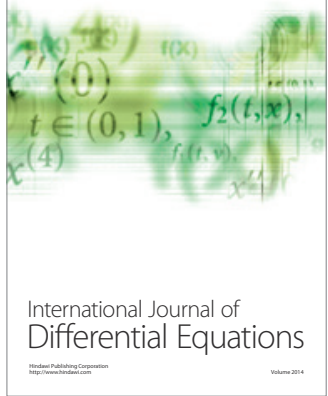
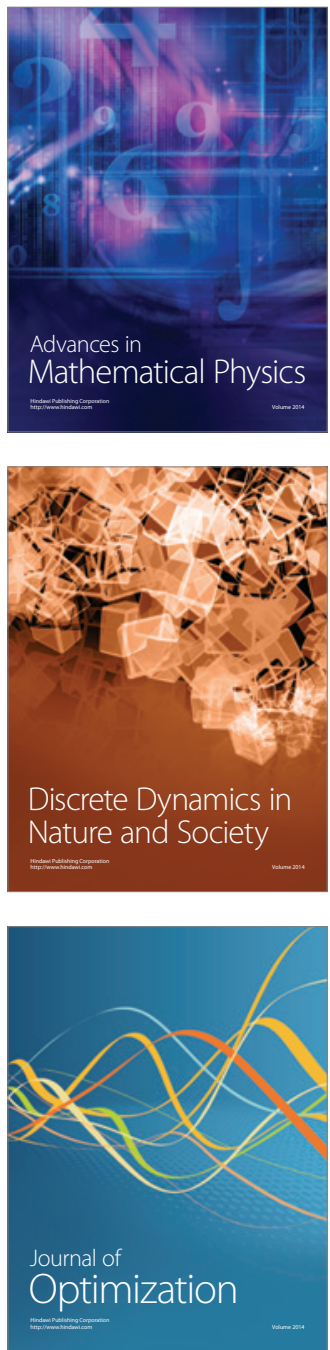